\title{
The approximate capacity for the 3 -receiver writing on random dirty paper channel
}

\author{
Stefano Rini * and Shlomo Shamai (Shitz) ${ }^{\dagger}$ \\ * National Chiao-Tung University, Hsinchu, Taiwan \\ E-mail: stefano@nctu.edu.tw \\ $\dagger$ Technion-Israel Institute of Technology, Haifa, Israel \\ E-mail: sshlomodee.technion.ac.il
}

\begin{abstract}
In this paper, the approximate capacity of the 3receiver "writing on random dirty paper" (WRDP) channel is derived. In the $M$-receiver WRDP channel, the channel output is obtained as the sum of the channel input, white Gaussian noise and a channel state sequence randomly selected among a set of $M$ independent Gaussian sequences. The transmitter has non-causal knowledge of the set of possible state sequences but does not know which one is selected to produce the channel output. In the following, we derive upper and lower bounds to the capacity of the 3-receiver WRDP channel which are to within a distance of at most 3 bits-per-channel-use (bpcu) for all channel parameters. In the achievability proof, the channel input is composed of the superposition of three codewords: the receiver opportunistically decodes a different set of codewords, depending on the variance of the channel state appearing in the channel output. Time-sharing among multiple transmission phases is employed to guarantee that transmitted message can be decoded regardless of the state realization. In the converse proof, we derive a novel outer bound which matches the pre-log coefficient arising in the achievability proof due to time-sharing. Although developed for the case of three possible state realizations, our results can be extended the general WRDP.
\end{abstract}

\section{INTRODUCTION}

The WRDP channel is the "writing on dirty paper" (WDP) channel [1] in which the channel state sequence is randomly selected among a set of independent Gaussian sequence. This model is equivalent to the compound channel in which the channel output at each receiver equals the sum of the channel input, Gaussian noise and a state sequence non-casually known at the transmitter. The WRDP channel models the scenario in which multiple known interferes can affect the transmission between a transmitter/receiver pair but the encoder does not know which interferer is active. In this paper, we consider the case of three possible state realizations and show that capacity can be approached by having the receiver opportunistically decode the superposition of different codewords, depending on the variance of the randomly selected channel state. Although we focus on the case of three possible independent state sequences, the bounding techniques we introduce here also extend to the WRDP channel with any number of state realizations.

The work of S. Rini was funded by the Ministry Of Science and Technology (MOST) under the grant 105-2221-E-009-029. The work of S. Shamai has been supported by the European Union's Horizon 2020 Research And Innovation Programme, grant agreement no. 694630.
Relevant Literature: In the Gelf'and-Pinsker (GP) channel [2], the channel output is a random function of the input and a channel state non-causally known at the transmitter. The compound extension of the GP channel has been investigated in [3], [4] where an achievable region for the two-receiver compound GP channel is derived. It is shown, in particular, that using a common message improves over extensions of the GP coding scheme in which the encoder performs joint binning for both receivers simultaneously. ${ }^{1}$ The additive Gaussian noise, additive Gaussian state version of the compound GP is first studied in [7] where this model is termed the "carbon copying onto dirty paper" (CCDP) channel. ${ }^{2}$ In [8] we study the CCDP in which states are a scaled version of a given sequence: for this model we show the approximate capacity in the "strong fading" parameter regime. In [9], we determine the approximate capacity of the CCDP with i.i.d. states (CCDPIS) channel, i.e. the CCDP channel in which the channel state sequences are independent and have equal variance.

Contributions: We determine the approximate capacity of the 3-receiver WRDP channel to within $3 \mathrm{bpcu}$ for all parameter regimes. The WRDP channel is a special case of the three-receive CCDP channel of [7] in which the channel state sequences are independent from each other. On the other hand, the WRDP channel generalizes the CCDP-IS channel of [9] as state sequences have any variance. In the achievability proof, the input is obtained as the superposition of three types of codewords: common, partially common and private codewords. Common codewords are decoded at all three receivers, partially common codewords at two receivers while private codewords only at one receiver. As different codewords are decoded by different sets of receivers, time-sharing among different transmission phases is employed to guarantee that all the receivers are able to decode the transmitted message. In the converse proof, we develop a new upper bound which recovers the pre-log coefficient arising in the achievable proof due to time-sharing.

Paper Organization: Sec. II introduces the channel model while Sec. III reviews the relevant results available in the literature. Sec. IV presents the main result. Finally, Sec. V concludes the paper.

\footnotetext{
${ }^{1}$ Note that the capacity for this model was incorrectly claimed in [5], [6].

${ }^{2}$ Although originally introduced for both the noiseless binary and the additive Gaussian compound GP channel, the term CCDP channel has generally been used to refer exclusively to the Gaussian case.
} 


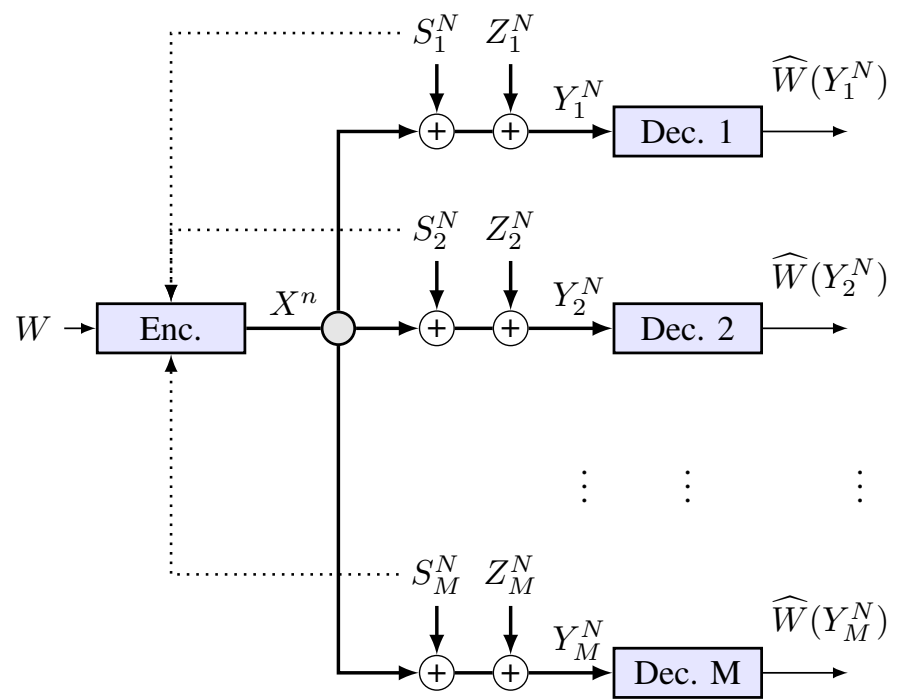

Fig. 1: The $M$-receiver carbon copying onto dirty paper (CCDP) channel.

\section{CHANNEL MODEL}

The $M$-receiver CCDP channel, also depicted in Fig. 1, is the $M$-receiver compound WDP channel in which the channel output at the $m^{\text {th }}$ receiver is obtained as

$$
Y_{m}^{N}=X^{N}+S_{m}^{N}+Z_{m}^{N},
$$

for $m \in[1 \ldots M]$ and where $X^{N}$ is the channel input, $S_{m}^{N}$ the state sequence and $Z_{m}^{N}$ a white Gaussian noise sequence with zero mean and unitary variance. The channel input $X^{N}$ is subject to the average power constraint $\sum_{i=1}^{N} \mathbb{E}\left[\left|X_{i}\right|^{2}\right] \leq$ $N P$. For each channel use $i \in[1, \ldots, N]$,

$$
\left[S_{1 i}, \ldots, S_{M i}\right] \sim \text { i.i.d. } \mathcal{N}\left(0, \Sigma_{S}\right) .
$$

The transmitter has full and anti-causal knowledge of the set of channel state sequences $\left[S_{1}^{N}, \ldots, S_{M}^{N}\right]$ and wished to reliably communicate the message $W \in\left[1, \ldots, 2^{N R}\right]$ to all the compound receivers through the channel input $X^{N}$. In turn, each compound receiver $m, m \in[1, \ldots, M]$ produces an estimate of the transmitted message from the channel output as $\widehat{W}\left(Y_{m}^{N}\right) \in\left[1, \ldots, 2^{N R}\right]$.

The $M$-receiver CCDP with i.i.d. states (CCDP-IS) channel is defined as the $M$-receiver CCDP channel in which the state covariance matrix $\Sigma_{S}$ in (2) is obtained as

$$
\Sigma_{S}=\operatorname{diag}([Q, \ldots, Q]),
$$

for $Q \in \mathbb{R}^{+}$and where $\operatorname{diag}(\mathbf{v})$ indicates the square matrix with the elements of the vector $\mathbf{v}$ on the diagonal and zero everywhere else.

The $M$-receiver "writing on random dirty paper" (WRDP) channel is the generalization of the $M$-receiver CCDP-IS channel in which

$$
\Sigma_{S}=\operatorname{diag}\left(\left[Q_{1}, \ldots, Q_{M}\right]\right),
$$

for $0 \leq Q_{1} \leq Q_{2} \leq \ldots \leq Q_{M}$ without loss of generality.

\section{RELATED RESULTS}

The authors of [7] derive the first inner and outer bound to the capacity of the 2-receiver CCDP channel. In [9], we improve on the outer bounds of [7] and show the approximate capacity for the CCDP-IS channel. Specializing the results of [7] to the 2-receiver CCDP-IS channel yields the following bounds.

Theorem III.1. Upper and lower bounds for the 2-receiver CCDP-IS channel[7, Th. 3, Th. 4]. Consider the 2-receiver CCDP-IS channel: the capacity of this model is upper bounded as

$$
\begin{aligned}
& \mathcal{C} \leq R^{\text {OUT }}= \\
& \begin{cases}\frac{1}{4} \log \left(\frac{1+P}{Q / 4+1}\right)+\frac{1}{4}\left(\frac{1+P+Q+2 \sqrt{P Q}}{Q / 4+1}\right) & Q \leq 4 \\
\frac{1}{4} \log (1+P)+\frac{1}{4} \log (1+P+Q+2 \sqrt{P Q}) & Q>4 \\
-\frac{1}{4} \log (Q) & \end{cases}
\end{aligned}
$$

and lower bounded as

$$
\begin{aligned}
& \mathcal{C} \geq R^{\mathrm{IN}}= \\
& \begin{cases}\frac{1}{2} \log \left(1+\frac{P}{Q / 2+1}\right) & Q / 2 \leq 1 \\
\frac{1}{2} \log \left(\frac{P+Q / 2+1}{Q}\right)+\frac{1}{4} \log \left(\frac{Q}{2}\right) & 1<Q / 2 \leq P+1 \\
\frac{1}{4} \log (P+1) & Q / 2>P+1\end{cases}
\end{aligned}
$$

In [9], we show that lower bound in (6) is to within a small gap from capacity. Additionally, we expand the result from 2 compound receiver to any number of receivers.

Theorem III.2. Approximate capacity for the $M$-receiver CCDP-IS channel [9, Th. IV.1]. Consider the $M$-receiver CCDP-IS channel: the capacity of this model is upper bounded as

$$
\begin{aligned}
& \mathcal{C} \leq R^{\text {OUT }}= \\
& \left\{\begin{array}{cl}
\frac{1}{2} \log \left(1+\frac{P}{1+Q}\right)+\frac{9}{4} & M-1 \geq Q \\
\frac{1}{2 M} \log (1+P) & M-1<Q \leq(M-1)(P+1) \\
+\frac{M-1}{2 M} \log (Q)+\frac{3}{2} & \\
\frac{1}{2 M} \log (1+P)+2 & Q>(M-1)(P+1)
\end{array}\right.
\end{aligned}
$$

and capacity is to within $2.25 \mathrm{bpcu}$ from the bound in (7).

In the attainable strategy for Th. III.2, the channel input is obtained as the superposition of two codewords: the base codeword treats the channel states as additional noise and is decoded at all receivers. The top codeword is pre-coded against the $m^{\text {th }}$ channel state for a portion $1 / M$ of the time. The converse proof is shown by establishing a recursion in the bounding of the capacity through a judicious choice of the side information at each compound receiver. The outer bound expression is further tightened using the fact that the capacity is non-increasing in the state variance.

The relatively simple expression of the result in Th. III. 2 is made possible by the assumption that the channel states have equal variance and thus treating the channel state as noise attains the same rate at all compound receivers. 


\section{MAIN RESULT}

In this section, we extend the result in Th. III.2 by deriving the approximate capacity of the 3-receiver WRDP channel.

In the achievability proof, the channel input is the superposition of three types of codewords: private, partially common and common codewords, each decoded at one, two and three receivers respectively. Since every compound receiver decodes a different set of codewords, time-sharing among different achievable strategies is used to convey the message to all receivers. In the converse proof, we bound the capacity of the compound model with a linear combination of the capacity of the channels between the transmitter and each of the compound receivers. The coefficients in the linear combination are carefully chosen so that, after the appropriate manipulations, it is possible to recover the time-sharing pre-log in the achievability proof.

The derivation of achievability and converse proofs can be extended beyond the case of three receivers: the difficulty in considering a more general case arises from the complexity of upper and lower bound expressions, as illustrated by the statement of the main theorem.

Theorem IV.1. Approximate capacity of the 3-receiver WRDP channel. Consider the 3-receiver WRDP channel: the capacity for this model is upper bounded as

$$
\begin{aligned}
& \mathcal{C} \leq R^{\text {OUT }}= \\
& \left\{\begin{array}{lr}
2 & \text { if } P \leq 2 \\
\frac{1}{2} \log (1+P) & \text { if } \quad Q_{2} \leq Q_{3} \leq 1 \\
\frac{1}{2} \log (1+P)-\frac{1}{4} \log \left(Q_{3}\right) & \text { if } \quad Q_{2} \leq 1<Q_{3} \leq P+1 \\
\frac{1}{4} \log (1+P)+\frac{1}{2} & \text { if } \quad Q_{2} \leq 1 \leq P+1<Q_{3} \\
\frac{1}{6} \log (1+P)+\frac{1}{2} & \text { if } 1 \leq P+1 \leq Q_{2} \leq Q_{3} \\
\frac{1}{2} \log (P+1)-\frac{2 \log (P+1)}{4 \log (P+1)-\log \left(Q_{2}\right)} \cdot \frac{1}{2} \log (P+1)+2 \\
\text { if } 1 \leq Q_{2}<P+1 \leq Q_{3} \\
\frac{1}{2} \log (P+1)-\frac{2 \log \left(Q_{3}\right)}{4 \log \left(Q_{3}\right)-\log \left(Q_{2}\right)} \cdot \frac{1}{2} \log \left(Q_{3}\right)+2 \\
\text { if } 1 \leq Q_{2} \leq Q_{3}<P+1
\end{array}\right.
\end{aligned}
$$

and the capacity is to within $3 \mathrm{bpcu}$ from the bound in (8).

Proof: For the sake of brevity, we only focus on the last two regimes in (8) as they require the most interesting derivation. A sketch of the proof for the other regimes is as follows:

- If $P \leq 2$ : the capacity is necessarily smaller than $2 \mathrm{bpcu}$ as the AWGN capacity is smaller than 2 bpcu.

- If $Q_{2} \leq Q_{3} \leq 1$ : as the state variance is smaller than the noise variance, treating the states as additional noise approaches the AWGN channel capacity to within $2 \mathrm{bpcu}$.

- If $Q_{2} \leq 1<Q_{3} \leq P+1$ or $Q_{2} \leq 1 \leq P+1<Q_{3}$ : the channel essentially behaves as the CCDP channel with $Q_{1}=$ $Q_{2}=1$ since the additive noise variance dominates the channel state variance at receiver 1 and 2 . The capacity for the latter model can be obtained as a variation of the result in Th. III.2 and is equal to the expression in (7) for $Q=Q_{3}$.

- If $1 \leq P+1 \leq Q_{2} \leq Q_{3}$ : The channel substantially resembles a 3-receiver CCDP-IS channel with $P+1 \leq Q_{1}=$ $Q_{2}=Q_{3}$. The approximated capacity, in this case, is again a variation of the result in Th. III.2.

The approximate capacity for the remaining parameters regimes in (8) is shown as follows.

Achievability: Consider the following random-coding achievable scheme: rate-split the message $W$ into three sub-messages $W_{c}, W_{p c}$ and $W_{p}$ and transmitt these sub-messages using codewords $U_{c}^{N}, U_{p c}^{N}$ and $U_{p}^{N}$ defined as:

- A common codeword $U_{c}^{N}$ with power $\alpha P$ and carrying the message $W_{c}$ at rate

$$
R_{c}=\frac{1}{2} \log \left(1+\frac{\alpha P}{Q_{3}+1+\bar{\alpha} P}\right) .
$$

This codeword is decoded at all receivers and treats the three state sequences and the remaining codewords as noise.

- A partially common codeword $U_{p c}^{N}$ with power $\bar{\alpha} \beta P$ and carrying the message $W_{p c}$ at rate

$$
R_{p c}=\frac{1}{2} \log \left(1+\frac{\bar{\alpha} \beta P}{Q_{2}+1+\bar{\alpha} \bar{\beta} P}\right) .
$$

This codeword decoded at receivers 1 and 2, is superimposed over $U_{c}^{N}$ and treats $S_{1}^{N}$ and $S_{2}^{N}$ and the private codewords (see (12)) as noise.

- Three private codewords $U_{i p}^{N}$ for $i \in\{1,2,3\}$ at power $\bar{\alpha} \bar{\beta} P$, carrying the message $W_{i p}$ for

$$
\begin{aligned}
& W_{1 p}=W_{2 p}=W_{p} \\
& W_{3 p}=\left[\begin{array}{ll}
W_{p} & W_{p c}
\end{array}\right],
\end{aligned}
$$

with rates

$$
R_{i p}=\frac{1}{2} \log (1+\bar{\alpha} \bar{\beta} P), \quad i \in\{1,2,3\} .
$$

The codeword $U_{i p}^{N}$ is pre-coded against the state sequence $S_{i}$ and decoded only at the receiver $i$. Note that, since receiver 3 does not decode $U_{p c}^{N}$, the assignment in (11) guarantees that all receivers are able to recover $W$. Time-sharing can be used to set $R_{1 p}=R_{2 p}=R_{p}$ and $R_{3 p}=R_{p}+R_{p c}$ : to this end consider the following three transmission phases:

- Phase 1: $U_{c}^{N} \rightarrow U_{p c}^{N} \rightarrow U_{1 p}^{N}$ and $S_{1}^{N} \rightarrow U_{1 p}^{N}$

- Phase 2: $U_{c}^{N} \rightarrow U_{p c}^{N} \rightarrow U_{2 p}^{N}$ and $S_{2}^{N} \rightarrow U_{2 p}^{N}$

- Phase 3: $U_{c}^{N} \rightarrow U_{3 p}^{N}$ and $S_{3}^{N} \rightarrow U_{3 p}^{N}$

where $U^{N} \rightarrow V^{N}$ indicates that $V^{N}$ is superimposed $U^{N}$ and $U^{N} \rightarrow V^{N}$ indicates that $V^{N}$ is binned against $U^{N}$ as in [10]. Let phase 3 have duration $\lambda N$ and phase 1 and 2 have each duration $\bar{\lambda} N / 2$ for $\bar{\lambda}=(1-\lambda)$ : by choosing

$$
\begin{aligned}
& \frac{\lambda}{2} \log (1+\bar{\alpha} P)= \\
& \frac{\bar{\lambda}}{2} \log \left(1+\frac{\beta \bar{\alpha} P}{Q_{2}^{2}+1+\bar{\beta} \bar{\alpha} P}\right)+\frac{\bar{\lambda}}{4} \log (1+\bar{\beta} \bar{\alpha} P),
\end{aligned}
$$

we have $R_{1 p}=R_{2 p}=R_{p}$ and $R_{3 p}=R_{p}+R_{p c}$ as desired. 
Let the power allocation parameters $\alpha$ and $\beta$ be chosen as

$$
\bar{\alpha}=\min \left\{\frac{Q_{3}}{P}, 1\right\}, \quad \bar{\beta}=\min \left\{\frac{Q_{2}}{\bar{\alpha} P}, 1\right\} .
$$

The choice of $\lambda$ which attains the equality in (13) for the assignment in (14), yields the lower bound

$$
R^{\mathrm{IN}}=\frac{2 \log (P+1)-\log \left(Q_{2}\right)}{4 \log (P+1)-\log \left(Q_{2}\right)} \frac{1}{2} \log (P+1)-\frac{1}{3},
$$

for $1 \leq Q_{2}<P+1 \leq Q_{3}$, and the lower bound

$$
\begin{aligned}
R^{\mathrm{IN}} & =\frac{1}{2} \log (P+1) \\
& -\frac{2 \log \left(Q_{3}\right)}{4 \log \left(Q_{3}\right)-\log \left(Q_{2}\right)} \frac{1}{2} \log \left(Q_{3}\right)-1,
\end{aligned}
$$

for $1 \leq Q_{2} \leq Q_{3}<P+1$.

Converse proof and approximate capacity: The converse proof focuses on deriving an outer bound to match the pre-log coefficients in (15) and (16).

- If $1 \leq Q_{2}<P+1 \leq Q_{3}$ : Define

$$
q=\frac{\log (P+1)}{\log Q_{2}}, \quad J=4 q-1, \quad K=2 q-1 .
$$

Using Fano's inequality and for $J$ and $K$ in (17), we write

$$
\begin{aligned}
& \begin{array}{l}
N(R-\epsilon) \\
\leq \min \left\{I\left(Y_{1}^{N}, W\right), I\left(Y_{2}^{N} ; W\right), I\left(Y_{3}^{N} ; W\right)\right\} \\
\leq \frac{1}{J}\left\{(J-K-1) I\left(Y_{1}^{N} ; W\right)+I\left(Y_{2}^{N} ; W\right)\right. \\
\left.\quad \quad+K I\left(Y_{3}^{N} ; W\right)\right\} \\
\leq \frac{N}{2 J}\left\{(J-K) \log 2 \pi e(P+1)+K \log 2 \pi e\left(Q_{3}\right)+2\right\} \\
\quad-\frac{J-K-1}{J} H\left(Y_{1}^{N} \mid W\right) \\
\quad-\frac{1}{J} I\left(Y_{2}^{N} \mid W\right)-\frac{K}{J} H\left(Y_{3}^{N} \mid W\right),
\end{array}
\end{aligned}
$$

where (18b) follows from the Gaussian maximizes entropy property and the fact that $Q_{2} \leq P+1 \leq Q_{3}$. Since $J-K-1=$ $K$ by definition, the negative entropy terms can be bounded as

$$
\begin{aligned}
- & (J-K-1) H\left(Y_{1}^{N} \mid W\right)-H\left(Y_{2}^{N} \mid W\right)-K H\left(Y_{3}^{N} \mid W\right) \\
\leq- & K\left\{H\left(Y_{1}^{N}, Y_{3}^{N} \mid W\right)\right\}-H\left(Y_{2}^{N} \mid W\right) \\
\leq- & K\left\{H\left(Y_{1}^{N}-Y_{3}^{N}, Y_{1}^{N}+Y_{3}^{N} \mid W\right)-2 N\right\} \\
& -H\left(Y_{2}^{N} \mid W\right) \\
\leq- & K\left\{H\left(S_{1}^{N}-S_{3}^{N}\right)+H\left(Y_{1}^{N}+Y_{3}^{N} \mid W, Y_{1}^{N}-Y_{3}^{N}\right)\right\} \\
& +2 K N-H\left(Y_{2}^{N} \mid W\right),
\end{aligned}
$$

where (19a) follows from the transformation $V_{1}^{N}=Y_{1}^{N}-Y_{3}^{N}$ and $V_{2}^{N}=Y_{1}^{N}+Y_{3}^{N}$ which has Jacobian $2 N$. Note now that

$$
-H\left(S_{1}^{N}-S_{3}^{N}\right) \leq \frac{N}{2} \log 2 \pi e Q_{3},
$$

while, since $K \geq 1$ by assumption as $P+1 \geq Q_{2}$, we have

$$
\begin{aligned}
& -K H\left(Y_{1}^{N}+Y_{3}^{N} \mid W, Y_{1}^{N}-Y_{3}^{N}\right)-H\left(Y_{2}^{N} \mid W\right) \\
& \leq-H\left(Y_{1}^{N}+Y_{3}^{N}, Y_{2}^{N} \mid W, Y_{1}^{N}-Y_{3}^{N}\right)
\end{aligned}
$$

$$
\begin{aligned}
\leq & -H\left(Y_{1}^{N}+Y_{3}^{N}-2 Y_{2}^{N}, Y_{2}^{N} \mid W, Y_{1}^{N}-Y_{3}^{N}\right) \\
\leq & H\left(S_{1}^{N}+S_{3}^{N}-2 S_{2}^{N} \mid S_{1}^{N}-S_{3}^{N}\right) \\
& -H\left(Y_{2}^{N} \mid W, Y_{1}^{N}-Y_{3}^{N}, Y_{1}^{N}+Y_{3}^{N}-2 Y_{2}^{N}\right) \\
\leq & -\frac{N}{2} \log 2 \pi Q_{3}-H\left(Z_{2}^{N} \mid Z_{1}^{N}-Z_{3}^{N}, Z_{1}^{N}+Z_{3}^{N}-2 Z_{2}^{N}\right) \\
\leq & -\frac{N}{2} \log 2 \pi Q_{3}+\frac{N}{2} \log 2 \pi e 3,
\end{aligned}
$$

where (21a) follows from the fact that the transformation has unitary Jacobian. Combining (19) and (21) in (18b) we finally obtain the upper bound

$$
R^{\mathrm{OUT}}=\frac{K+1}{J} \log (P+1)-\frac{1}{J} \log \left(Q_{3}\right)+\frac{2 K+1}{J} .
$$

Note that the channel in which $Q_{3}=P+1$ has a larger capacity that the channel under consideration since part of the state $S_{3}^{N}$ can be provided to receiver 3 as side information. For this reason, substituting $Q_{3}$ with $P+1$ yields an upper bound to capacity. With this substitution, we obtain the expression in (22) which is to within $3 \mathrm{bpcu}$ from the expression in (15).

- If $1 \leq Q_{2} \leq Q_{3}<P+1$ : The converse proof for this regime is a variation of the proof for the previous regime: let

$$
q=\frac{\log Q_{3}}{\log Q_{2}}, \quad J=4 q-1, \quad K=2 q-1 .
$$

Repeating the derivation in (18a) for the values of $J$ and $K$ in (23), instead of (17), yields the bound

$$
\begin{aligned}
& N(R-\epsilon) \leq \frac{1}{2} \log 2 \pi e(P+1) \\
& -\frac{1}{2 J}\left\{(J-K-1) H\left(Y_{1}^{N} \mid W\right)+H\left(Y_{2}^{N} \mid W\right)\right. \\
& \left.\quad+K H\left(Y_{3}^{N} \mid W\right)\right\} .
\end{aligned}
$$

A bounding similar to (21) for the negative entropy terms of (24) yields

$$
\begin{aligned}
& -(J-K-1) H\left(Y_{1}^{N} \mid W\right)-H\left(Y_{2}^{N} \mid W\right)-K H\left(Y_{3}^{N} \mid W\right) \\
& \leq-K H\left(S_{1}^{N}-S_{3}^{N}\right) \\
& \quad-H\left(S_{1}^{N}+S_{3}^{N}-2 S_{2}^{N} \mid S_{1}^{N}-S_{3}^{N}\right),
\end{aligned}
$$

so that combining (24) and (25) we obtain the upper bound

$$
R^{\text {OUT }}=\frac{1}{2} \log (P)-\frac{K+1}{J} \log \left(Q_{3}\right)+2,
$$

which is to within 3 bpcu from the lower bound in (16).

We next provide few remarks on the result in Th. IV.1.

Remark IV.2. $M$-receiver CCDP-IS channel. When the channel states have the same variance, as Th. III.2, partially common do not provide any rate improvement. Treating the channel state as additional noise attains the same rate for all compound receivers: thus only private and common codewords are necessary to approach capacity.

Remark IV.3. Extensions to the $M$-receiver WRDP channel. The result in Th. IV.1 can be extended to the case of multiple receivers as follows. In the lower bound, $M-2$ partially common codeword can be constructed for the subset of receivers $[1, \ldots, m]$ with $m \in[2, M-1]$. Accordingly, $M$ transmission phases can be used to guarantee that all the receivers are 
able to decode all the sub-messages. In the converse proof, the time-sharing coefficients in the achievable strategy can be used to bound Fano's inequality for the compound channel as in (18) and (24). Unfortunately, the approximate capacity expression contains a number of sub-cases that are not easily manipulated algebraically. For this reason, we are currently unable to provide a sufficiently compact representation of the capacity in the general case.

Remark IV.4. Time-sharing VS superposition coding. The achievable strategy in the proof of Th. IV.1 can be improved upon by using code-sharing instead of time-sharing as follows:

$$
\begin{aligned}
& U_{c}^{N} \rightarrow U_{p c}^{N}, U_{p c}^{N} \rightarrow U_{1 p}^{N}, U_{p c}^{N} \rightarrow U_{2 p}^{N}, \\
& U_{c}^{N} \rightarrow U_{3 p}^{N}, U_{p c}^{N} \rightarrow U_{3 p}^{N}, \\
& S_{1}^{N} \rightarrow U_{1 p}^{N}, S_{2}^{N} \rightarrow U_{2 p}^{N}, S_{3}^{N} \rightarrow U_{3 p}^{N}, \\
& U_{1 p}^{N} \ldots U_{2 p}^{N}, U_{1 p}^{N} \cdots U_{3 p}^{N}, U_{2 p}^{N} \cdots U_{3 p}^{N},
\end{aligned}
$$

where we have used the notation of [10]. The achievable strategy in (27) provides a bounded performance improvement over the time-sharing strategy used in the achievability proof of Th. IV.1 On the other hand, the simpler transmission strategy employed in Th. IV.1 can be more easily optimized over the transmission parameters as in (10) and compared to the outer bound expression.

Remark IV.5. Non-unique decoding. Indirect or non-unique decoding as in [4] is not necessary for the result in Th. IV.1. As argued in [11], joint (unique) decoding is sufficient to approach capacity to within a small gap.

The result in Th. IV. 1 is plotted in Fig. 2 for the case in which $Q_{1}, Q_{2}$ and $Q_{3}$ are kept fixed while $P$ grows. Let $\mathcal{R}_{[a, b]}$ indicate the upper bound region of (8) for $P \in[a, b]$, then: in $\mathcal{R}_{[0,2]}$ the capacity is upper bounded by a constant since the channel input power is smaller the additive noise variance. In the region $\mathcal{R}_{\left[2, Q_{2}\right]}$, the channel state variance dominates the transmit power and only private codewords can be transmitted reliably. For $\mathcal{R}_{\left[Q_{2}, Q_{3}\right]}$, the transmitter uses a partially common codeword to simultaneously communicate to receiver 1 and 2 while receiver 3 only receives a private codeword. In $\mathcal{R}_{\left[Q_{3}, \infty\right]}$ the transmitter can use both a common codeword to communicate to all the receivers as well as a partially common codeword for receiver 1 and 2 .

The result in Th. IV.1 also provides the generalized degrees of freedom of the 3-receiver WRDP channel.

Lemma IV.6. Generalized degrees of freedom. Let $Q_{2}=$ $P^{\alpha}$ and $Q_{3}=P^{\beta}$, for $\alpha \leq \beta$, then the generalized degrees of freedom of the 3-receiver WRDP channel are obtained as

$$
\lim _{P \rightarrow \infty} \frac{\mathcal{C}(P)}{\log (P)}= \begin{cases}\frac{1}{2} & \alpha=0, \beta=0 \\ \frac{1}{2}-\frac{1}{4} \beta & \alpha=0, \beta<1 \\ \frac{1}{4} & \alpha=0, \beta \geq 1 \\ \frac{1}{2}-\frac{\alpha}{4-\alpha} & \alpha \leq 1 \leq \beta \\ \frac{1}{6} & 1 \leq \alpha \leq \beta \\ \frac{1}{2}-\frac{2 \beta}{4 \beta-\alpha} \beta & \alpha \leq \beta \leq 1\end{cases}
$$

\section{CONCLUSION}

This paper studies a variation of the "writing on dirty paper" channel in which the channel state is randomly drawn

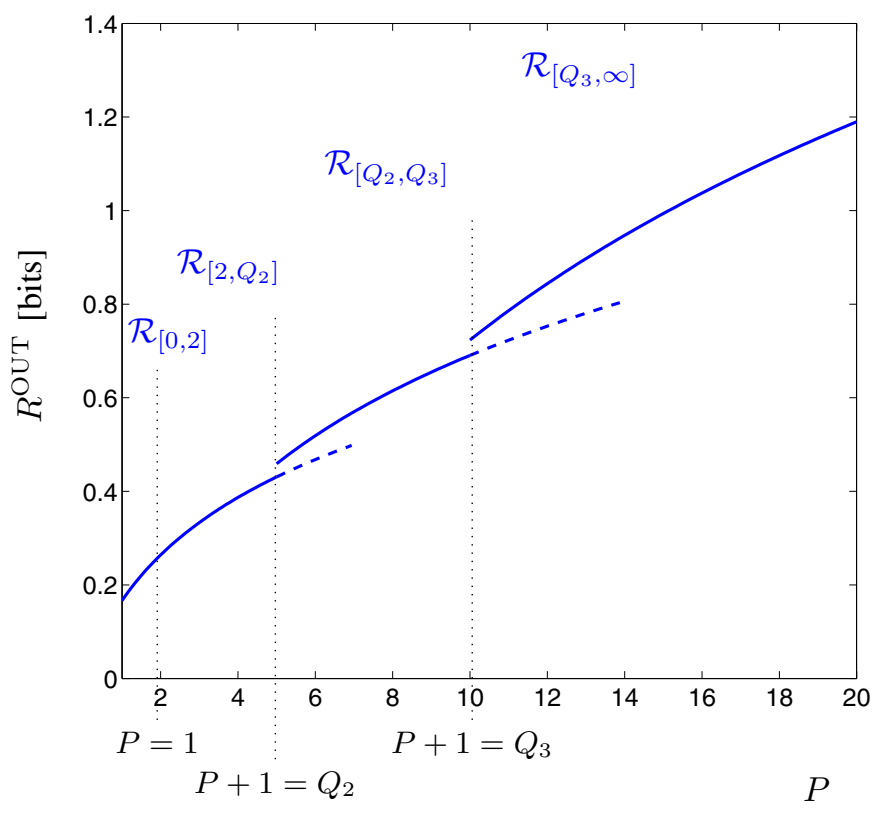

Fig. 2: A plot of the upper bound in Th. IV.1 for $Q_{1}=0$, $Q_{2}=5 Q_{3}=10$ and $P \in[1,20]$.

among three possible independent Gaussian realizations. For this model, capacity can be approached to within $3 \mathrm{bpcu}$ by the use of a partially common codeword, that is codeword that is decoded at two of the three receivers. A matching converse is obtained by bounding the capacity with a carefully-chosen linear combination of the capacity of the channel toward each of the compound receivers.

\section{REFERENCES}

[1] M. Costa, "Writing on dirty paper." IEEE Trans. Inf. Theory, vol. 29, no. 3, pp. 439-441, 1983.

[2] S. Gel' fand and M. Pinsker, "Coding for channel with random parameters," Probl. Contr. Inform. Theory, vol. 9, no. 1, pp. 19-31, 1980.

[3] P. Piantanida and S. Shamai, "On the capacity of compound statedependent channels with states known at the transmitter," in 2010 IEEE International Symposium on Information Theory, June 2010, pp. 624628.

[4] C. Nair, A. E. Gamal, and Y.-K. Chia, "An achievability scheme for the compound channel with state noncausally available at the encoder," arXiv preprint arXiv:1004.3427, 2010.

[5] P. Piantanida and S. Shamai, "Capacity of compound state-dependent channels with states known at the transmitter," in 2009 IEEE International Symposium on Information Theory. IEEE, 2009, pp. 1968-1972.

[6] P. Moulin and Y. Wang, "Capacity and random-coding exponents for channel coding with side information," IEEE Transactions on Information Theory, vol. 53, no. 4, pp. 1326-1347, 2007.

[7] A. Khisti, U. Erez, A. Lapidoth, and G. Wornell, "Carbon copying onto dirty paper," IEEE Trans. Inf. Theory, vol. 53, no. 5, pp. 1814-1827, May 2007.

[8] S. Rini and S. Shamai, "On capacity of the dirty paper channel with fading dirt in the strong fading regime," in Information Theory Workshop (ITW), 2014 IEEE. IEEE, 2014, pp. 561-565.

[9] - "On the capacity of the carbon copying onto dirty paper channel," International Conference on the Science of Electrical Engineering in Israel (ICSEE), 2016.

[10] S. Rini and A. Goldsmith, "A unified graphical approach to random coding for single-hop networks," IEEE Transactions on Information Theory, vol. 62, no. 1, pp. 56-88, 2016.

[11] S. S. Bidokhti and V. M. Prabhakaran, "Is non-unique decoding necessary?" IEEE Transactions on Information Theory, vol. 60, no. 5, pp. 2594-2610, 2014. 